\title{
Performance Analysis of Large Intelligent Reflecting Surface Aided Moderate MIMO for 5G Communication
}

Fitsum Dessalegn Mekonnen

Addis Ababa Science and Technology University

Muluneh Mekonnen Tulu ( $\nabla$ mulumoke@yahoo.com )

Addis Ababa Science and Technology University https://orcid.org/0000-0002-2420-525X

Sultan Feisso

Addis Ababa Science and Technology University

\section{Research Article}

Keywords: 5G, IRS, ADMM, MIMO, SINR

Posted Date: October 20th, 2021

DOI: https://doi.org/10.21203/rs.3.rs-826873/v1

License: (c) (i) This work is licensed under a Creative Commons Attribution 4.0 International License.

Read Full License 


\title{
Performance Analysis of Large Intelligent Reflecting Surface Aided Moderate MIMO for 5G Communication
}

\author{
Fitsum Dessalegn Mekonnen \\ Muluneh Mekonnen Tulu and \\ Sultan Feisso
}

Received: date / Accepted: date

\begin{abstract}
The recently completed 5G framework is the outcome of a few advanced technologies. Massive Multiple Input Multiple Output (MIMO), millimeter wave communication, and network densification are examples of these technologies. However, there are two disadvantages to this technology.1) the lack of control over the wireless channel, and 2) the wireless interface's excessive power consumption. The concept of re-configurable Intelligent Reflecting Surfaces has emerged to answer the need for green and cost-effective future cellular networks. In this study, we'll look at how using an Intelligent Reflecting Surface (IRS) improve the performance of moderate MIMO communication in terms of the rate, SINR, energy efficiency and transmit power metrics. Despite the fact that the underlying issue is non-convexity, we use Lagrangian dual transform and Quadratic Transform to change and rearrange the original issue. After that, active and passive beam forming improved alternatively using an alternating Direction method of multiplier algorithm (ADMM.The IRS-aided

Fitsum Dessalegn Mekonnen

Department of Electrical \& Computer Engineering, Addis Ababa Science and Technology University, Addis Ababa, Ethiopia.

Tel.: +251-923242006

E-mail: dfitsum06@gmail.com

Muluneh Mekonnen Tulu

Tel.: +251-913123353

E-mail: mulumoke@yahoo.com

Department of Electrical \& Computer Engineering, Addis Ababa Science and Technology University, Addis Ababa, Ethiopia.

Artificial \& Robotics Intelligence, COE, Addis Ababa Science and Technology University, Addis Ababa, Ethiopia.

Sultan Feisso

Tel.: +251-912635020

E-mail: sultan.feisso@aastu.edu.et

Department of Electrical \& Computer Engineering, Addis Ababa Science and Technology University, Addis Ababa, Ethiopia.

Artificial \& Robotics Intelligence, COE, Addis Ababa Science and Technology University, Addis Ababa, Ethiopia.
\end{abstract}


system with a reasonable number of antennas at the access point (AP) outperforms the massive MIMO without IRS in terms of sum rate, SINR, energy efficiency and transmit power metrics.

Keywords $5 \mathrm{G} \cdot \mathrm{IRS} \cdot \mathrm{ADMM} \cdot \mathrm{MIMO} \cdot \mathrm{SINR}$

\section{Introduction}

Through many creative developments, such as massive (MIMO), millimeter wave (mm Wave) communication, and network densification, the Fifth Generation (5G) network standard promises to deliver enhanced bandwidth, accessibility, and incredibly low delay. Regardless of this advancement, providing services of consistent quality to clients in difficult propagation environments consumes a significant amount of power. The total network energy consumption, for instance, is related to the number of base stations (BS) and active antenna elements at each BS. Communication in the $\mathrm{mm}$ Wave bands suffers from significant path/penetration losses. As a result of these issues, future cellular networks must be green and sustainable, with control over the propagation environment. The smart radio environment (SRE) is a new concept that fulfills this demand by changing the wireless propagation environment into an intelligent tunable space that engages in radio frequency transmission from sender to receiver [1]. This concept can be realized by placing low-cost antenna arrays [2], smart reflect-arrays [3], and re-configurable meta-surfaces in the environment to passively shape intruding electromagnetic (EM) waves in the desired direction without producing additional radio signals. In this paper, we will see the importance of Intelligent Reflecting Surfaces to improve the performance of moderate MIMO system.

\subsection{Related Works}

In [4], the comparative analysis of intelligent Reflecting surfaces (IRS's) as well as amplify and forward (AF) relaying wireless networks Considered, and it is demonstrated that IRS aided wireless systems has a higher spectral efficiency (SE) than the corresponding AF relaying wireless systems. In [5], a survey conducted on the IRS is presented this encompasses IRS-aided communications data rate and capacity assessments, deep learning-based design, power/spectral optimization's, and reliability analysis they also look into IRS deployments and how IRS's can be used for secure communication, terminal location, and other novel applications. The author in [6], investigate the multi user down-link MISO system, which is helped by the IRS. To optimize the WSR under the BS transmit power constraint, a hybrid active and passive beam forming issue is defined. An iterative solution based on the recently announced fractional programming methodology has been designed to solve this non convex issue. Three low-complexity algorithms are also offered to solve the problem. In [7], massive MIMO designs for milli meter wave (mm Wave) communication is proposed It is shown that properly locating the active receiving antennas for the intelligent surface significantly improves the spectral efficiency of RIS supported MIMO 
structures. Furthermore, they advocate for a power usage framework for RIS supported radio network that accounts for RIS effect imperfections, such as taper loss, spillover loss, phase shifter loss and aperture loss. In [8], the use of re-configurable intelligent surface (RIS) to enhance uplink beam formation gain in a massive MIMO system is investigated. It has also been demonstrated that IRS can improve network throughput. In [9], Performance analysis of Re-configurable intelligent surface (RIS) in multi-user MIMO systems was outlined. The capacity of both of the up link and down link channels has been discussed. In [10], after the concentrated weighted sum rate augmentation problem has been transformed to a manageable sub problem, the author presents an incremental alternate direction of multiplier (ADMM) calculation to up-date the beam former locally. The author in [11], investigated the problem of "capacity-maximization for MIMO IRS-assisted point-to-point communication, using joint IRS reflection coefficients". An alternative optimization strategy was proposed for frequency-flat channels, which provides a locally optimized solution by optimizing a single optimization variable ("the transmit co-variance matrix"). In [12], an IRS-assisted (MISO) remote framework was presented to consider where an IRS is sent to help a single radio wire client to receive the correspondence from the multiple receiving wire. So, the customer receives the channel directly from the AP at the same time, just as the IRS does. The author in [13], propose a twofold IRSassisted multiple input multiple output (MIMO) communication framework for LOS channels. Advance the transmit co-variance network and the inactive beam forming grids of the two agreeable IRSs to investigate the limit amplification issue. The author in [14], offered three analytic approaches to play out the finding in a variety of settings with varying IRS's CSI requirements and computing complexities. In [15], the use of an IRS at the cell limit improved the cell-edge client execution of multi cell communication frameworks. They addressed the WSR augmentation problem by simplifying the passive shifts at the IRSs and Active Transmit Precoding (TPC) lattices at the AP, while ensuring that each BS's power requirement and unit-modulus limitation at the IRS were met. In [16], in order to deal with inaccurate CSI estimates, the author devised as well as solved "a robust probabilistic constrained optimization problem for IRS-aided MISO communication systems. The best beam forming vector there at BS as well as reflecting elements at the IRS are determined iteratively with the converging alternative optimization approach.

\section{System Model}

\subsection{Down-link Moderate MIMO System with IRS Support}

We consider an Intelligent Reflecting Surface (IRS) supported Moderate MIMO communication framework as displayed in figure 1 in which there is a BS furnished with 'N' Transmitting Antennas, M' Reflecting passive components Serving 'K' numerous antenna users each of has ' $Q$ ' Antenna components Serving ' $K$ ' numerous antenna users each of has ' $Q$ ' Antenna components where $((N>1, Q>=1)$ indicate by $S=\left[S 1, \ldots . S_{k}\right]^{T} \in C^{k \times Q}$ and 'the Gaussian information images in which every component is a free arbitrary variable with zero mean and unit variance, likewise $S_{k}$ 
component image sent to the $K^{\text {th }}$ client. An IRS element is thought to be with Max elements on a level plane and May elements in an upward direction.( $\mathrm{M}=$ Max by May). The smart controller also controlled the IRS unit, which arranges the reflecting

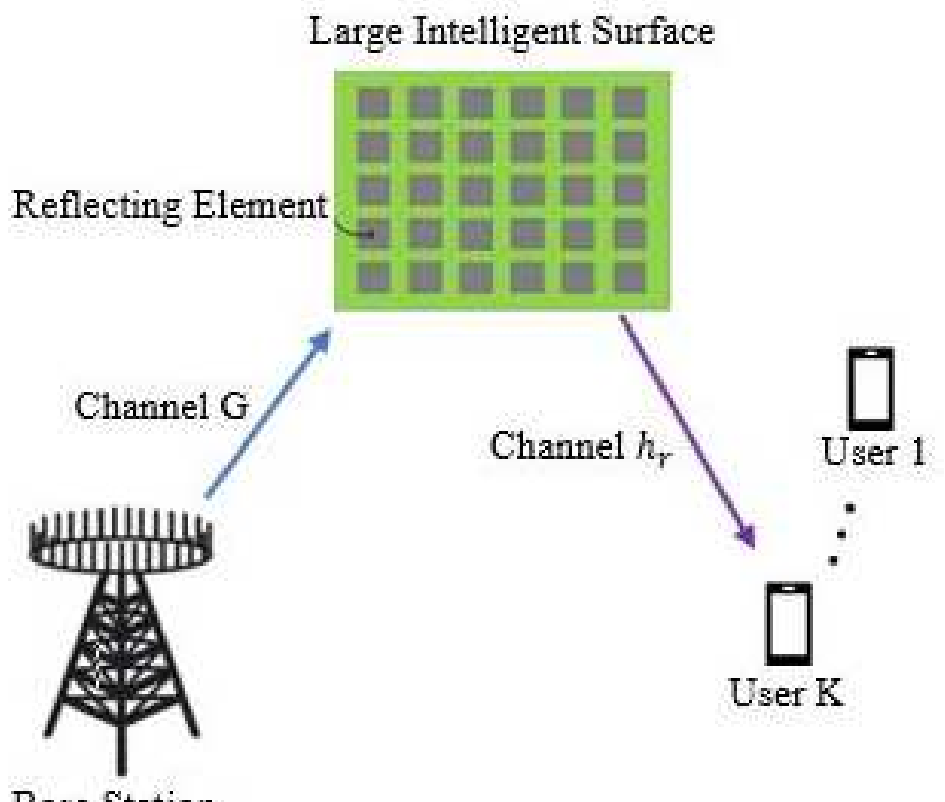

\section{Base Station}

Fig. 1 IRS aided Moderate MIMO Communication System [17]

modes for The IRS unit. Where $w_{k} \in C^{M \times K}$ is the matrix of Linear Transmit Precoding (TPC) used by BS to send its information vector $S_{k}$ to the $k^{\text {th }}$ user. Also, Base band channels crossing through BS to IRS, and those from IRS to the $K^{\text {th }}$ user, are denoted by $G \in C^{N \times M}$ and $h_{r, k} \in C^{M \times 1}$ in the order given. We assume that the BS has "Perfect channel - state information (CSI)" [18] for all channels involved and all the channels are quasi-Static flat-fading Rayleigh. In likewise, we Denote $\theta_{n} \in F$ the RC of the $n^{\text {th }}$ reflection component, where $F$ is the feasible set of RCs.

The BS transmits a signal that is given by:

$x=\sum_{k=1}^{k} w_{k} s_{k}$

We consider the following assumption for the feasible set of RCs: $\phi=\operatorname{diag}\left(\beta \theta_{1} \ldots \beta \theta_{n}\right)$ ,$\theta_{n} \in[0,2 \pi]$ and $\beta \in[0,1]$ are the diagonal phase-shift lattice for the IRS, the combined incident signal's phase shift and amplitude reflection coefficient respectively. Now let's defined the total transmitted signal by: 
$Y_{k}=h_{r, k}{ }^{H} \phi G X+n_{k}$

Where $n_{k}=C N\left(0, \sigma^{2}\right)$ represents additive white Gaussian noise (AWGN) at the $K^{t h}$ user receiver.Then by substituting (2.1) in to (2.2) we get

$Y_{k}=\left(h_{r, k}{ }^{H} \phi G\right) \sum_{k=1}^{K} w_{k} s_{k}+n_{k}$

\subsection{Problem Formulation}

All signals from other users are treated as interference by the $K^{\text {th }}$ user. As a result,

$$
y_{k}=\frac{\left|\left(h_{r, k}{ }^{H} \phi G\right) w_{k}\right|^{2}}{\sum_{i=1, i \neq k}^{k}\left|\left(h_{r, k}{ }^{H} \phi G\right) w_{i}\right|^{2}+\sigma^{2}}
$$

Where $w_{k}=\left[w_{1}, w_{2} \ldots \ldots . w_{k}\right] \in C^{M \times k}$ and the major goal is to increase the WSR by creating the transmit beam forming matrix $\mathrm{W}$ at the $\mathrm{BS}$ and the phase shift matrix Phi at the IRS together. The Spectral Efficiency (SE) (bits/Hz) at user k is given by:

$S E=R_{K}(W, \phi)=\log _{2}\left(1+y_{k}\right)$

And the Sum rate is given by:

$S R=S_{\text {sum }}(W, \phi)=\sum_{k=1}^{K} w_{k} R_{k}(w, \phi)$

Therefore, the WSR maximization problem is become

$$
\begin{array}{ll}
\text { (P1) } & \max _{w, \phi} f_{1}(w, \phi)=\sum_{k=1}^{K} w_{k} \log _{2}\left(1+y_{k}\right) \\
\text { s.t } \quad & \sum_{k=1}^{K}\left\|w_{k}\right\|^{2} \leq P_{\max }(t) \\
& \theta_{n} \in F, \forall_{n}=1, \ldots \ldots, M
\end{array}
$$

2.3 Beam forming Design with Active and Passive Components

First, we have to make decouple the optimization of TPC matrix W and the phase shift matrix $\phi$ in to several tractable sub problems. So by using Lagrangian dual transform we can address the logarithm problem in the objective function of (P1). Hence, we use the proposed Lagrangian dual transform in [19] then (P1) becomes in the form,

$\left(P 1^{*}\right) \max _{w, \phi} f_{2}(w, \phi, \alpha)$ 
s.t $\quad \sum_{k=1}^{K}\left\|w_{k}\right\|^{2} \leq P_{\max }(t)$
$\theta_{n} \in F, \forall_{n}=1, \ldots \ldots, M$

Where $\alpha$ refers to $\left[\alpha_{1}, \alpha_{2}, \ldots \ldots \ldots \alpha_{k}\right]^{T}$ is an extra vector introduced by the Lagrangian dual transform. The WSR of Problem (2.7) can be written as:

$f_{2}(W, \phi, \alpha)=\sum_{k=1}^{K} w_{k} \log _{2}\left(1+\alpha_{k}\right)-\sum_{k=1}^{K} w_{k} \alpha_{k}+\sum_{k=1}^{K} \frac{w_{k}\left(1+\alpha_{k}\right) y_{k}}{1+y_{k}}$

Thus solving (2.7) is the same as solving (2.9) so, we provide a solution (2.9) by using an alternating iterative method that yields three alternating steps i.e., the auxiliary variable $\alpha$, Active Beam Forming and Passive Beam Forming. So, in ( $\left.\mathrm{Pl}^{*}\right)$ when w and $\phi$ hold fixed and setting $\frac{d f_{2}}{d \alpha_{k}}=0$ The optimal $\alpha$ is:

$\alpha_{k}^{0}=y_{k}$

Then for affixed $\alpha$ optimizing $\mathrm{W}$ and $\phi$ is reduced to:

$$
\begin{aligned}
& \left(P 1^{\text {new }}\right) \max _{w, \phi} \sum_{k=1}^{K} \frac{\overline{\alpha_{k}} y_{k}}{1+y_{k}} \\
& \text { s.t } \quad \sum_{k=1}^{K}\left\|w_{k}\right\|^{2} \leq P_{\max }(t) \\
& \quad \theta_{n} \in F, \forall_{n}=1, \ldots \ldots, M
\end{aligned}
$$

Here, $\overline{\alpha_{k}}=w_{k}\left(1+\alpha_{k}\right)$ Since, $\mathrm{W}$ and $\theta$ are related only to the SINR term the other variables in (2.9) will be ignored.Then we are going solve ' $\mathrm{W}$ ' by holding $\phi$ and solve $\phi$ by holding ' $\mathrm{W}$ ' respectively.

\subsection{Active Beam forming scheme}

Let us first optimize The ABF by fixing the PBF matrix. For Problem 2.11 let denote the combined channel for user $\mathrm{k}$ by:

$h_{k}=h_{r, k}{ }^{H} \phi G X$

Then the SINR in (2.4) becomes:

$y_{k}=\frac{\left|h_{k}{ }^{H} w_{k}\right|^{2}}{\sum_{i=1, i \neq k}^{k}\left|h_{k}{ }^{H} w_{i}\right|^{2}+\sigma^{2}}$

So,by using 2.13 the primary function of problem 2.11 can be expressed as a function of 'W'.

$f_{3}(w)=\sum_{k=1}^{K} \frac{\overline{\alpha_{k}} y_{k}}{1+y_{k}}$ 
Substituting (2.13) in to (2.14) we get:

$\sum_{k=1}^{K} \frac{\overline{\alpha_{k}}\left|h_{k}{ }^{H} w_{k}\right|^{2}}{\sum_{i=1, i \neq k}^{k}\left|h_{k}{ }^{H} w_{i}\right|^{2}+\sigma^{2}}$

Given this ' $\alpha$ ' and ' $\phi$ ' enhancing ' $W$ ' becomes:

$(P 2) \max _{w} f_{3}(w)$

s.t $\quad \sum_{k=1}^{K}\left\|w_{k}\right\|^{2} \leq P_{\max }(t)$

$\theta_{n} \in F, \forall_{n}=1, \ldots \ldots, M$

We not that (2.14) is a fractional programming problem with multiple ratios. As a result, by employing the quadratic transform proposed technique in [19] we can write (2.15) as:

$f_{4}(w, \beta)=\sum_{k=1}^{k} 2 \sqrt{\overline{\alpha_{k}}} \operatorname{Re}\left\{\beta_{k} h_{k}{ }^{H} w_{k}\right\}-\sum_{k=1}^{K}\left|\beta_{k}\right|^{2} \sum_{i=1}^{k}\left(\left|h_{k}{ }^{H} w_{i}\right|^{2}+\sigma^{2}\right)$

Where, $\beta=\left[\beta_{1}, \ldots . \beta_{k}\right]^{T}$ is the additional variable introduced by using the quadratic transform. and (2.17) is a problem of biconvex enhancement.we can rewrite 2.17 as:

$f(w, \theta, \alpha, \beta)=\sum_{k=1}^{K} 2 \sqrt{\overline{\alpha_{k}}} \operatorname{Re}\left\{\beta_{k} h_{k}{ }^{H} w_{k}\right\}-\sum_{k=1}^{K}\left|\beta_{k}\right|^{2}\left(\sum_{i=1}^{k}\left|h_{k}{ }^{H} w_{i}\right|^{2}+\sigma^{2}\right)$

The ADMM method is then used to solve problem (P2) perfectly [20]. Problem (P2) augmented 's Lagrangian is:

$f(w, \theta, \alpha, \beta, \lambda)=f(w, \theta, \alpha, \beta)+\left(\sum_{k=1}^{K}\left\|w_{k}\right\|^{2}-P_{t}\right)+\sum_{n=1}^{N} I F\left(\theta_{n}\right)+\frac{p}{2}\left\|\theta_{n}+\lambda\right\|_{2}^{2}$

Then the variables can be updated incrementally by:

$$
\begin{aligned}
& \alpha^{i_{0}+1}=\arg \max L\left(\theta^{i_{0}}, w^{i_{0}}, \alpha, \beta^{i_{0}}, \lambda^{i_{0}}\right) \\
& \beta^{i_{0}+1}=\arg \max L\left(\theta^{i_{0}}, w^{i_{0}}, \alpha^{i_{0}+1}, \beta, \lambda^{i_{0}}\right) \\
& w^{i_{0}+1}=\arg \max L\left(\theta^{i_{0}}, w, \alpha^{i_{0}+1}, \beta^{i_{0}+1}, \lambda^{i_{0}}\right) \\
& \theta^{i_{0}+1}=\arg \max L\left(\theta, w^{i_{0}+1}, \alpha^{i_{0}+1}, \beta^{i_{0}+1}, \lambda^{i_{0}}\right) \\
& \lambda^{i_{0}+1}=\lambda^{i_{0}}+P\left(\theta^{i_{0}+1}\right)
\end{aligned}
$$

The optimal solution of $\alpha$ for problem (2.20a) is

$\alpha^{0}=y_{k}$ 
This is obtained when $\mathrm{W}$ and $\theta$ hold fixed. The optimal solution of $\beta$ for problem (2.20b) is:

$\beta^{0}=\frac{\sqrt{\overline{\alpha_{k}}} h_{k}{ }^{H} w_{k}}{\sum_{i=1, i \neq k}^{K}\left|h_{k}{ }^{H} w_{i}\right|^{2}+\sigma^{2}}$

This is obtained by setting $d f_{4} / d \beta_{k}=0$ The optimal solution of $\mathrm{W}$ for problem (2.20c) is:

$w_{k}{ }^{0}=\sqrt{\overline{\alpha_{k}}} \beta_{k}\left(\lambda_{0} I_{N}+\sum_{i=1}^{k}\left|\beta_{i}\right|^{2} h_{i} h_{i}{ }^{H}\right)^{-1} h_{k}{ }^{H}$

This is obtained by setting $d f_{4} / d w_{k}=0$ Where $\lambda_{0} \geq 0$ is the best dual variable to use in the transmit power constraint

$\lambda_{0}=\min \left\{\lambda_{0} \geq 0: \sum_{k=1}^{K}\left\|w_{k}\right\|^{2} \leq P \max (t)\right\}$

\subsection{Passive Beam forming scheme}

To find the optimal solution of $\theta$ we first rearrange some equations with some mathematical manipulation so,(2.15) can be re written as:

$h_{k}{ }^{H} w=\theta^{H} \operatorname{diagh}_{r, k}{ }^{H} G W$

Where

$\phi^{H}=\theta^{H} \operatorname{diagh}_{r, k}{ }^{H}$

To simplify let;

$v_{k, j}=\operatorname{diagh}_{r, k}{ }^{H} G W$

Then we rewrite (2.19) as:

$\max f_{5}(\theta)=\sum_{k=1}^{K} \frac{\overline{\alpha_{k}}\left|\theta^{H} v_{k, k}\right|^{2}}{\sum_{i=1, i \neq k}^{k}\left|\theta^{H} v_{k, j}\right|^{2}+\sigma^{2}}$
s.t $\quad 0 \leq \theta_{n} \leq 2 \pi, \forall_{n}=1, \ldots \ldots, M$

Then using QT- we can write(2.25) as

$\max f_{6}(\theta, r)=\sum_{k=1}^{K} 2 \sqrt{\overline{\alpha_{k}}} \operatorname{Re}\left\{r_{k} \theta^{H} v_{k, k}\right\}-\left|r_{k}^{2}\right|\left(\sum_{i=1}^{k}\left|\theta^{H} v_{k, j}\right|^{2}+\sigma^{2}\right)$ 
Where ' $r$ ' is the auxiliary variable introduced by the Lagrange multiplier-based Quadratic Transform, and setting $d f_{6}(\theta, r) / d r_{k}$ the optimal ' $r$ ' is given by:

$r_{k}^{0}=\frac{\sqrt{\alpha_{k}} \theta^{H} v_{k, k}}{\sum_{j=1}^{k}\left|\theta^{H} v_{k, j}\right|^{2}+\sigma^{2}}$

Given ' $r$ ' the optimization of $\theta$ can be written as:

$\max f_{7}(\theta, r)=-\theta^{H} A \theta+2 \operatorname{Re}\left\{\theta^{H} b\right\}-\sum_{k=1}^{K} r_{k}^{2} \sigma^{2}$

Where

$\left[\mathrm{A}=\sum_{k=1}^{K}\left|r_{k}^{0}\right|^{2} \sum_{j=1}^{K} v_{k, j} v_{k, j}\right.$

and

$\left[\mathrm{b}=\sum_{k=1}^{K} \sqrt{\overline{\alpha_{k}}} r_{k}^{0} v_{k, k}\right.$

Then, by removing the constant terms that are unrelated to $\theta$, we can rewrite:

$\max f_{8}(\theta, r)=-\theta^{H} A \theta+2 \operatorname{Re}\left\{\theta^{H} b\right\}$

[s.t $0 \leq \theta_{n} \leq 2 \pi, \forall_{n}=1, \ldots, M(2.29 b)$

Problem (2.29) is an example of a convex optimization problem. The Lagrangian dual decomposition method can be used to solve (2.29). Lagrange's dual of (2.29) can be expressed as [21]:

$\underset{\lambda}{\min i \operatorname{imize}} L(\lambda)=\max _{\theta} G(\theta, \lambda)$

s.t $\lambda_{n} \geq 0, \forall_{n}=1, \ldots \ldots, M$

where $\lambda=\left[\lambda_{1}, \ldots \ldots \ldots \lambda_{n}\right]$ is the dual variable for the constraint $\theta^{H} e_{n} e_{n} \theta \leq 1$ and $G(\theta, \lambda)$ denotes the dual objective function given by:

$G(\theta, \lambda)=f_{8}(\theta)-\sum_{n=1}^{N} \lambda_{n}\left(\theta^{H} e_{n} e_{n} \theta-1\right)$

Where $e_{n} \in R^{m \times 1}$ primary level vector with a one in the $k^{\text {th }}$ position and zeros everywhere else. Then by setting $d G / d \theta=0$ The optimal $\theta$ can be obtained as:

$\theta^{0}=\left(A+\sum_{k=1}^{M} \lambda_{n} e_{n} e_{n}{ }^{H}\right)^{-1} \quad b=D(\lambda) b$ 
Table 1 Simulations and Algorithmic Parameters

\begin{tabular}{ll}
\hline Parameters & Values \\
\hline Algorithm and convergence parameter & 0.01 \\
Transmission bandwidth BW & $20 \mathrm{MHz}$ \\
Maximum transmit Power at the BS & $30 \mathrm{dBm}$ \\
AWGN & $-85 \mathrm{dBm}$ \\
\hline
\end{tabular}

Utilizing Schur complement technique proposed in [22], the problem in (2.30) could be designed as a problem of Semi Definite Programming(SDP).

$\underset{\varepsilon \lambda}{\max i m i z e \varepsilon}-\operatorname{tr}(\operatorname{diag}(\lambda))$

s.t $\left[\begin{array}{cc}A+\operatorname{tr}(\operatorname{diag}(\lambda)) & b \\ b^{H} & -\varepsilon\end{array}\right] \geq 0$

Where $D(\lambda)=(A+\operatorname{diag}(\lambda))^{-1}$ So, by solving (2.33) using CVX tool box we get the optimal value of $\lambda$

\section{Algorithm}

[1. Input: $\mathrm{G}, \mathrm{h}_{r,}, v_{k}$

[2. Output: $\mathrm{w}, \theta$, Sum_rate

[3. $\mathrm{i}_{0} \leftarrow 0$

[4. While stopping criterion not satisfied do

[5. Update $\alpha^{i_{0}+1}, \forall k$ using (2.21)

[6. Update $\beta^{i_{0}+1}, \forall k$ using (2.22)

[7. Update $\mathrm{w}^{i_{0}+1}, \forall k$ using $(2.23)$

[8. Update $\theta^{i_{0}+1}$, byusing $(2.20 e)$

[9. Update $\lambda^{i_{0}+1}$, byusing $(2.20 f)$

[10. With given $\theta$ update $\phi$

[11. $\mathrm{i}_{0} \leftarrow i_{0}+1$

[12. Until the Stopping Criterion is Met.

[13. end while

\section{Results and Discussions}

\subsection{Introduction}

In this section, simulation results and corresponding analysis are presented following the Algorithm analysis discussed in section 2. Simulation results are carried out to compare the performance of massive MIMO and IRS aided Moderate MIMO systems 


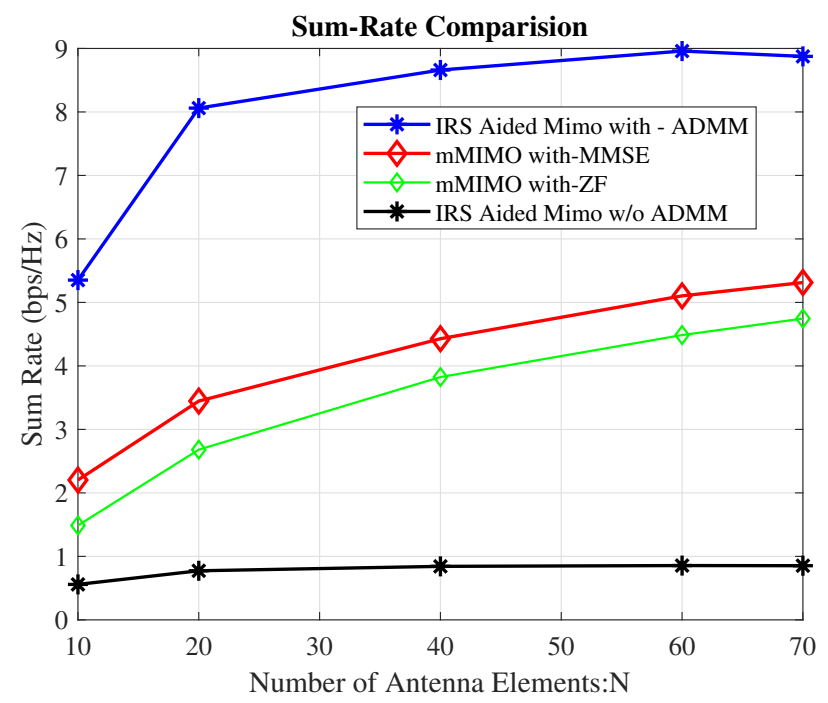

Fig. 2 A comparison of the four schemes' sum rates.

\subsection{Performance Metrics}

Performance measures such as Sum Rate, SINR, Energy efficiency and Transmit Power are used to analyze and validate the proposed system.

\subsection{Sum Rate}

One of the measures used to compare the performance of massive MIMO and IRSassisted moderate MIMO communication systems is the user sum rate. So, to compare the network throughput achieved by the four techniques below, we present a numerical example: IRS-assisted MIMO communication with and without the ADMM optimization algorithm and massive MIMO with MMSE and ZF beamforming algorithm without IRS. We assume the BS is installed with $\mathrm{N}=70$ antennas, $\mathrm{K}=3$ users, and the number of IRS elements varies between 8 and 80 with five number of points. Other important parameters are available in table 1.Fig. 2 illustrates the sum-rate performance of the three users characterized by various IRS element counts under the four schemes. Initially, it is noticed that the sum-rate performance of the IRS-assisted MIMO system is poorer than that of the massive MIMO system without IRS. Next, it is observed that under the optimal ADMM beamforming method described in $(2.23$ and 2.28), The sum-rate obtained in the IRS- assisted MIMO system is significantly higher than that obtained in the massive MIMO system without IRS. 


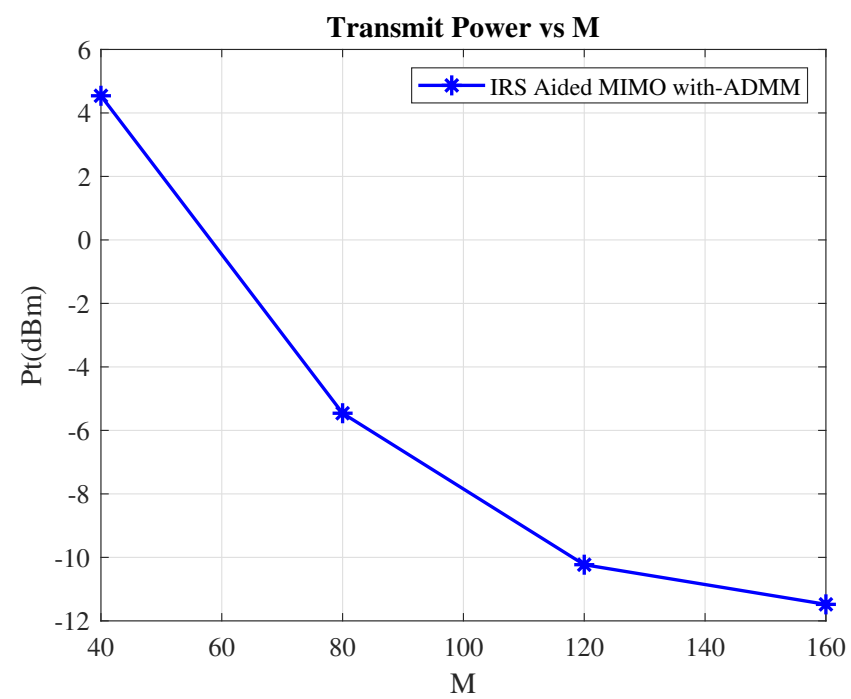

Fig. 3 Transmit power vs M

\subsection{Transmit Power}

As shown in Figure 3, The overall transmit power is reduced by using large intelligent reflecting surfaces. So, there is a significant power saving with IRS. For example, with 40 number of Reflecting elements the Transmitted power is $4.5 \mathrm{dBm}$ and when we increase the number of IRS elements to 60 the transmitted power is dropped to $2 \mathrm{dBm}$ and as we continually increase the number of reflecting elements to 160 the transmitted power will drop to around $-11 \mathrm{dBm}$.

\subsection{SINR}

The other metrics used to compare massive MIMO and IRS-assisted moderate MIMO communication systems is SINR. So, we examine the network SINR achieved by the following Three schemes: $\mathrm{N}=40$ with $\mathrm{M}=80, \mathrm{~N}=40$, and $\mathrm{N}=70$, total amount of antenna elements at the BS and the total amount of reflecting elements at the IRS, respectively, are $\mathrm{N}$ and $\mathrm{M}$. The number of Users $\mathrm{K}=20$. So, as we can see in figure 4 Passive IRS elements helps to reduce the number of active antennas (see $\mathrm{N}=40$ with $\mathrm{M}=80$ vs $\mathrm{N}=40$ and $\mathrm{N}=80$ without IRS).

\subsection{Energy Efficiency}

Another criterion used to compare the performance of IRS-aided moderate MIMO versus massive MIMO without the IRS is energy efficiency. as we can see in figure 5 IRS aided communication is critical to create energy efficient wireless system.(where $\mathrm{K}=20, \mathrm{~N}=70$ and $\mathrm{M}=120$ ). 


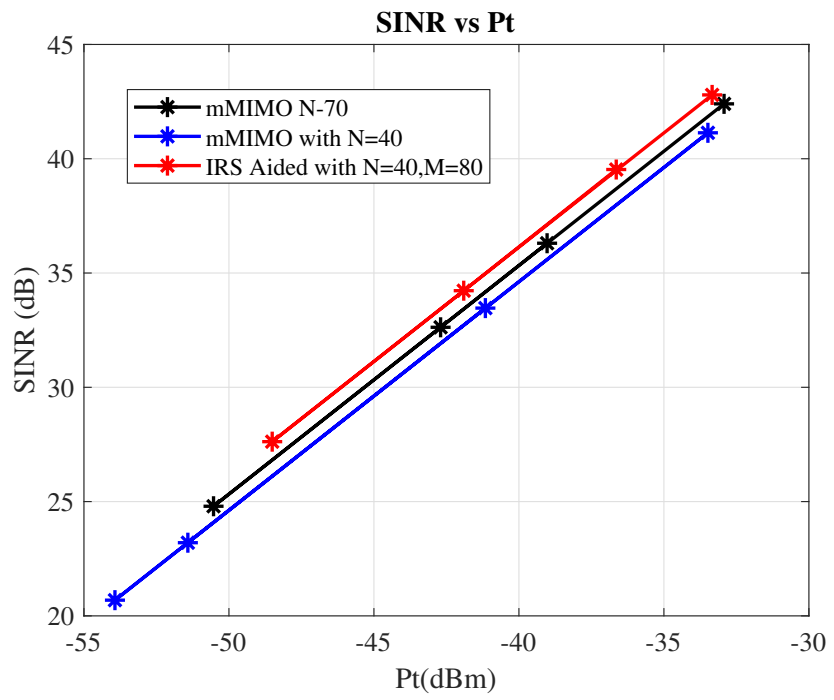

Fig. 4 SINR vs Transmit power

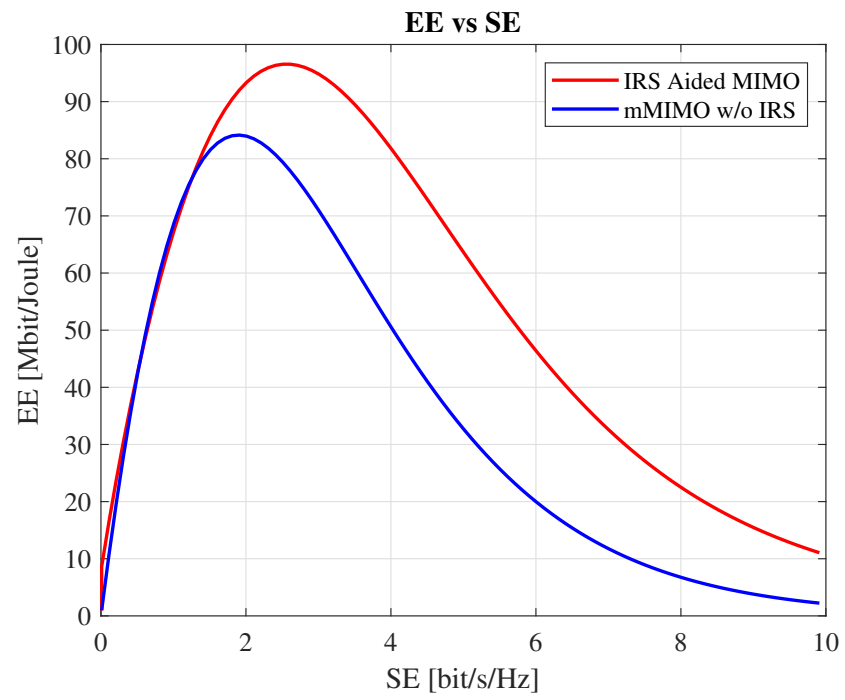

Fig. 5 Energy Efficiency vs Spectral Efficiency

\section{Conclusion}

In this paper we have suggested the IRSs to enhance the performance of massive MIMO system. Specifically, to enhance the user rate, SINR,Energy efficiency and lowering the overall transmit power at the access point are all goals. The IRS aided system does not have satisfactory result compared to massive MIMO system if it has not optimized by a proper beam forming algorithm. We have two different beam- 
forming schemes the first is active beam forming at the AP, and the second is inactive reflecting beam forming at the IRS. So, we have proposed an ADMM algorithm to jointly optimize the two beam forming schemes. We demonstrated, using this solution, that the IRS-aided moderate MIMO system achieve higher user rate, SINRs, energy efficiency and low transmit power compared to its counterpart without IRS. Because of this, IRS aided MIMO has proven to be very effective in improving the efficiency of massive MIMO system.

\section{Declaration}

Currently there is no any fund support this research work.Also,there is no conflicts of interest/Competing interests within the authors. In addition,the data sets generated during/or analyzed during the current study are available from the corresponding author on reasonable request.

\section{References}

1. Marco Di Renzo, Merouane Debbah,Dinh-Thuy Phan-Huy,Zappone,Mohamed-SlimAlouini,Chau Yuen, Vincenzo Sciancalepore, George C. Alexandropoulos,Jakob Hoydis,Haris,Gacanin,Julien de Rosny, Ahcene Bounceu, Geoffroy Lerosey and Mathias Fink, "Smart Radio Environments Empowered by AI Reconfigurable Meta-Surfaces:An Idea Whose Time Has Come," http://arxiv.org/abs/1903.08925[Cs.IT], vol. 1, pp. 3-8, 1 April 2019.

2. Manideep Dunna, Chi Zhang, Daniel Sievenpiper, Dinesh Bharadia., "ScatterMIMO: Enabling Virtual MIMO with Smart Surfaces," In The 26th Annual International Conference on Mobile Computing and Networking (MobiCom '20), September 21-25, 2020, London, United Kingdom ACM, New York, NY, USA, 14 pages. https://doi.org/10.1145/3372224.3380887, vol. 1, pp. 3-7, September 2020.

3. Xin Tan, Zhi Sun, Dimitrios Koutsonikolas, "Enabling Indoor Mobile Millimeter-wave Networks," IEEE INFOCOM 2018 - IEEE Conference on Computer Communications, vol. 1,pp. 1-6,2018.

4. Alexandros-Apostolos A. Boulogeorgos and Angeliki Alexiou,'Performance Analysis of Reconfigurable Intelligent Surface-Assisted Wireless Systems and Comparison With Relaying,' IEEE Access, vol. 8, no. Received May 7,pp. 2-7, June 2, 2020.

5. Jun Zhao and Yang Liu, "A Survey of Intelligent Reflecting Surfaces (IRSs):Towards 6G Wireless Communication Networks," http://arxiv.org/abs/1907.04789, vol. 3,pp. 5-7,12 December 2019.

6. Huayan Guo, Ying-Chang Liang,Jie Chen, and Erik G. Larsson, "Weighted Sum-Rate Optimization for Intelligent Reflecting Surface Enhanced Wireless Networks," http://arxiv.org/abs/1905.07920, vol. 2,pp. 1-12, 30 May,2019.

7. Vahid Jamali, Antonia M. Tulino, Georg Fischer, Ralf Muller, and Robert Schober, 'Intelligent Reflecting and Transmitting Surface Aided Millimeter Wave Massive MIMO," arXiv:1902.07670 [cs.IT] , vol. 2,pp. 6, 23 Sep 2019.

8. Zhaorui Wang, Liang Liu and Shuguang Cui, "Intelligent Reflecting Surface Assisted Massive MIMO Communications," arXiv:2002.05899 [cs.IT] , vol. 3,pp. 5-9, 5 May 2020.

9. S. Liu, "Performance Analysis of Intelligent Reflecting Surface in Multi-user MIMO Systems," J. Phys.: Conf. Ser. 1575 012078,pp. 3-6 2020.

10. S. Huang, "Rate, Reliability and Secrecy Performance Analysis and Optimization for Millimeter Wave Communications," Doctoral Thesis, pp. 39-44, Stockholm, Sweden 2020.

11. Shuowen Zhang and Rui Zhang, "Capacity Characterization for Intelligent Reflecting Surface Aided MIMO Communication,” arXiv:1910.01573 [cs.IT] , vol. 1,pp. 4-6, 3 Oct 2019.

12. Qingqing Wu and Rui Zhang, ”Intelligent Reflecting Surface Enhanced Wireless Network: Joint Active and Passive Beamforming Design," http://arxiv.org/abs/1809.01423, vol. 1,pp. 2-4, 5 Sep 2018.

13. Yitao Han, Shuowen Zhang, Lingjie Duan and Rui Zhang, "Double-IRS Aided MIMO Communication under LoS Channels: Capacity Maximization and Scaling," arXiv:2102.13537 [cs.IT] , vol. 1,pp. 2-7, 26 Feb 2021. 
14. Rui Sun, Weidong Wang, Li Chen, Guo Wei, and Wenyi Zhang, "Diagnosis of Intelligent Reflecting Surface in Millimeter-wave Communication Systems," arXiv:2101.03792 [eess.SY] , vol. 1,pp. 3-7, 11 Jan 2021.

15. Cunhua Pan, Hong Ren, Kezhi Wang, Wei Xu, Maged Elkashlan, Arumugam Nallanathan and Lajos Hanzo, "Multicell MIMO Communications Relying on Intelligent Reflecting Surfaces," arXiv:1907.10864 [eess.SP], vol. 4,pp. 5-7, 24 Apr 2020.

16. Tuan Anh Le, Trinh van Chien and Marco Di Renzo, "Robust Probabilistic-Constrained Optimization for IRS-Aided MISO Communication Systems," IEEE wireless communications letters, IEEE comsoc, In press, ff10.1109/LWC, vol. 1, 23 Nov 2020.

17. Chongwen Huang,Alessio Zappone,George C. Alexandropoulos, M'erouane Debbah and Chau Yuen, "Reconfigurable Intelligent Surface: Energy Efficiency and Intelligent Configuration in Wireless Communication," IEEE VTS and Comsoc Joint Workshop on 6G SUTD, p.14, Apr 24, 2019.

18. Fakoorian, Ali Swindlehurst, A., "On the Optimality of Linear Precoding for Secrecy in the MIMO Broadcast Channel," Selected Areas in Communications, IEEE Journal on, vol. 31, p. 9, 2013/09/01.

19. Kaiming Shen and Wei Yu, "Fractional Programming for Communication Systems-Part II: Uplink Scheduling via Matching," IEEE Transactions on Signal Processing., pp. 2-3, Jan 9, 2017.

20. J. Romberg., "Alternating direction method of multipliers (ADMM)," Georgia Tech ECE 8823a, vol. 1, pp. 2-6, March 10, 2017.

21. P. Parrilo and S.Lall, "onvexity and Duality," CDC 2003, vol. 3, p. 21, 2003.12.07.

22. Kolapo Alli, IAENG, A.M Jubril, and L.O Kehinde, ”Development of a Semi-definite Programming Weighted Sum Based Approach for Solving Stochastic Multi-objective Economic Dispatch Problems Incorporating CHP Units," IAENG International Journal of Computer Science, vol. 4, p. 36, 20 November 2017. 